Portland State University

PDXScholar

Electrical and Computer Engineering Faculty

Publications and Presentations

Electrical and Computer Engineering

4-1-1977

\title{
Single particle scattering with focused laser beams
}

Lee W. Casperson

Portland State University

Cavour W. Yeh

Follow this and additional works at: https://pdxscholar.library.pdx.edu/ece_fac

Part of the Electrical and Computer Engineering Commons

Let us know how access to this document benefits you.

\section{Citation Details}

Lee W. Casperson, C. Yeh, and Wing F. Yeung, "Single particle scattering with focused laser beams," Appl. Opt. 16, 1104-1107 (1977).

This Article is brought to you for free and open access. It has been accepted for inclusion in Electrical and Computer Engineering Faculty Publications and Presentations by an authorized administrator of PDXScholar. Please contact us if we can make this document more accessible: pdxscholar@pdx.edu. 


\title{
Single particle scattering with focused laser beams
}

\author{
Lee W. Casperson, C. Yeh, and Wing F. Yeung
}

\begin{abstract}
A scattering technique is described in which the incident laser beam is tightly focused to isolate the effects of a single particle. In this way the individual particles may be studied in their natural environment, and experiments with latex spheres are in agreement with the theory.
\end{abstract}

\section{Introduction}

The importance of using light scattering techniques to determine the physical properties of particulates is well recognized. ${ }^{1-3}$ Optical methods may be fast, inexpensive, and nondestructive, and under optimum conditions the individual particles can be studied in surroundings approaching their natural environment. When the scattering objects are known to be nearly identical (monodisperse) and spherically symmetric, substantial amounts of information can be obtained from the spatial distribution of light scattered from several particles simultaneously. However, for critical applications where the size or shape of individual particles must be determined within a polydisperse medi$\mathrm{um}$, it is essential that in some way the effects of the particles be isolated. A brute force method for accomplishing this isolation involves electrostatically supporting an individual particle while it is illuminated by a laser beam. ${ }^{4}$ The simplest application of single particle scattering is in particle counting and sizing. For this purpose gas suspended particles may be made to flow through a small illuminated aperture one at a time, and the resulting flashes of light provide information on the density and size of the particles.

In the previously mentioned single-particle scattering schemes the environment of the scatterer is somewhat artificial. This fact may be of great consequence when one deals with biologically interesting objects such as viruses and bacteria and when one wishes to obtain more precise information on pollutants, since the mere fact of isolation is likely to alter the properties of the species under study. The purpose of this work is to describe a new type of scattering technique which is designed to overcome some of these limitations. The general experimental configuration is shown schematically in Fig. 1. An incident laser beam is tightly fo-

\footnotetext{
The authors are with University of California, School of Engineering \& Applied Science, Los Angeles, California 90024.

Received 19 August 1976.
}

cused by means of lenses to yield a focal spot in the scattering material with a diameter on the order of the wavelength of the light. If the beam is kept stationary it will, from time to time, impinge on one of the scatterers which drifts into the focal region. When this occurs, there will be a pulse of scattered light which can be detected to yield information about that specific scatterer. An advantage of this technique is that in many cases a polydisperse ensemble can be studied particle by particle without removing the scatterers from their normal surroundings.

\section{Concentration Limits}

As a first step in the analysis we estimate the maximum particle concentration for which this technique is useful. The incident beam is assumed to have the complex Gaussian amplitude distribution

$$
\begin{aligned}
E(r, z)=E_{o}\left(w_{o} / w\right) \exp -i[k z+ & k r^{2} / 2 R \\
& \left.-i r^{2} / w^{2}-\tan ^{-1}\left(z \lambda / \pi w_{o}{ }^{2}\right)\right],
\end{aligned}
$$

where $w$ is the $1 / e$ spot size, $R$ is the radius of curvature of the spherical phase fronts, and $\lambda$ is the wavelength in the medium. The spot size varies with $z$ according to

$$
w=w_{o}\left[1+\left(\lambda z / \pi w_{0}{ }^{2}\right)^{2}\right]^{1 / 2},
$$

where $w_{o}$ is the spot size at the beam waist. The light will be strongly scattered by any particles located within the spot radius, and the scattering will be relatively unimportant for particles outside of this radius. Also, the intensity falls off rapidly for distances $z$ away from the beam waist. If we define $z_{m}$ as the maximum distance at which scattering is important, the total active volume can be found from the integral

$$
\begin{gathered}
V=\pi \int_{-z_{m}}^{z_{m}} w^{2}(z) d z=\pi w_{o}{ }^{2} \int_{-z_{m}}^{z_{m}}\left[1+\left(\lambda z / \pi w_{o}{ }^{2}\right)^{2}\right] d z \\
=\pi w_{0}{ }^{2}\left[2 z_{m}+2 z_{m}{ }^{3}\left(\lambda / \pi w_{0}{ }^{2}\right)^{2} / 3\right] .
\end{gathered}
$$

At best one might expect to eliminate from the scattering data events which occur where the intensity is down to half of its maximum value. From Eq. (2) this occurs at the Rayleigh length $z_{o}=\pi w_{o}^{2} / \lambda$, and Eq. (3) reduces to $V=8 \pi^{2} w_{o}^{4} / 3 \lambda$. If the data analysis proce- 


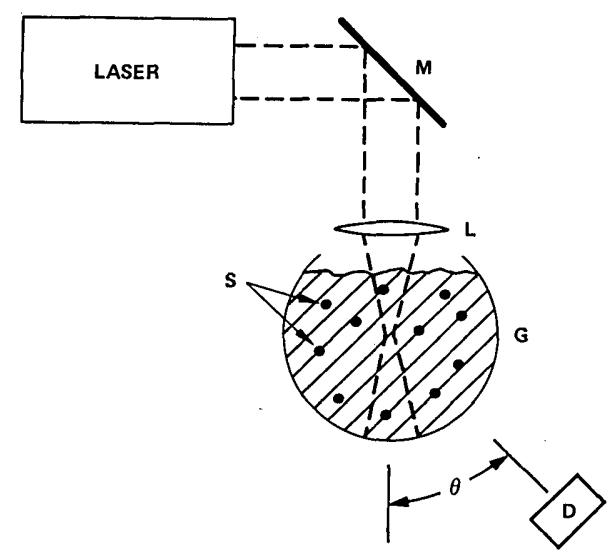

Fig. 1. Schematic drawing of experimental apparatus (not to scale) used in focused beam scattering studies with $M$, a plane mirror; $L$, a microscope objective lens; $G$, a spherical glass envelope containing the scatterers; $D$, a low noise detector; and $S$, individual scatterers.

dure can tolerate an average of one particle in this volume, the maximum usable particle density is $\rho_{m}=$ $3 \lambda / 8 \pi^{2} w_{0}{ }^{4}$. Thus the maximum particle density depends critically on the minimum spot size $w_{o}$, and it is essential to use high quality low $f$-number optics. From Eq. (2) the far-field diffraction angle is $w / z=\lambda / \pi w_{0}$. But the maximum diffraction angle associated with a lens is approximately $(2 f)^{-1}$, where $f$ is the $f$-number. Therefore, the minimum spot size can be written $w_{o}=$ $2 \lambda f / \pi$, and the maximum density is

$$
\rho_{m}=\frac{3 \pi^{2}}{128 \lambda^{3} f^{4}}=\frac{0.23}{\lambda^{3} f^{4}} .
$$

For an $f$ value of unity and a wavelength of $5000 \AA$ the maximum density is $1.85 \times 10^{12} \mathrm{~cm}^{-3}$. If one uses a lens having a larger $f$, or if a smaller number of particle coincidences is required, this maximum concentration would be reduced accordingly. There is no minimum usable density, but with low particle concentrations, data accumulation will, of course, be very slow.

\section{Particle Motion}

Another aspect of the focused beam scattering technique involves motion of the scattering object. Any object suspended in a gas or liquid exhibits a random drifting behavior known as Brownian motion. This motion is helpful in that it continuously brings new particles into the beam. On the other hand, without feedback and beam tracking methods the Brownian motion places a lower limit on the residence time of each particle in the beam and imposes a limit on the response time of the data recording system.

The distribution function for a particle in a fluid can be written

$$
f(\bar{r}, t)=(4 \pi D t)^{-3 / 2} \exp \left(\frac{-\left|\bar{r}-\bar{r}_{o}\right|^{2}}{4 D t}\right),
$$

where $\bar{r}_{o}$ is the initial position vector of the particle at time $t=0$, and $D$ is the diffusion coefficient. ${ }^{5}$ For a spherical particle of radius $a$ in a fluid of viscosity $\eta$ the diffusion coefficient is

$$
D=k T / 6 \pi a \eta,
$$

where $k$ is Boltzmann's constant and $T$ is the temperature. The average time spent by a particle within the beam can be estimated from Eq. (5) by assuming that the particle starts at the axis of the cylindrical beam volume at time $t=0$. Then, one can calculate the time at which the integral of the distribution function over the beam volume is equal to one half, or

$$
\begin{gathered}
\frac{1}{2}=(4 \pi D t)^{-3 / 2} \int_{0}^{2 \pi} \int_{0}^{\omega_{0}} \int_{-\infty}^{\infty} \exp \left[-\frac{\left(r^{2}+z^{2}\right)}{4 D t}\right] d z d r d \theta \\
=1-\exp \left(-w_{0}{ }^{2} / 4 D t\right)
\end{gathered}
$$

Therefore, the interaction time is about

$$
t_{\mathrm{I} / 2}=\frac{w_{0}{ }^{2}}{4 D \ln 2}=\frac{\lambda^{2} f^{2}}{\pi^{2} D \ln 2} .
$$

To illustrate the application of these formulas we consider an example corresponding to the experiment described later in this paper. The viscosity of water at $25^{\circ} \mathrm{C}$ is approximately $0.90 \mathrm{cP}$ or $0.90 \times 10^{-3} \mathrm{~N}$-sec $/ \mathrm{m}^{2}{ }^{6}$ Therefore, from Eq. (6) a particle of radius $a=0.2405$ $\mu \mathrm{m}$ will have a diffusion coefficient $D=1.01 \times 10^{-12}$ $\mathrm{m}^{2} / \mathrm{sec}$. The wavelength of the incident beam is $\lambda=$ $0.5145 \mu \mathrm{m}$, and the focusing lens has an $f$-number of 1.65 corresponding to a beam waist spot size $w_{0}=2 \lambda f / \pi=$ $0.54 \mu \mathrm{m}$. Thus Eq. (8) implies that the residence time of a particle near the focus is about $t_{1 / 2}=0.1 \mathrm{sec}$. This value agrees with typical pulse lengths observed in our experiments. For very large values of focal intensity the above analysis can be generalized to include the effects of radiation pressure.

\section{Scattered Field Distribution}

In the previous sections a general focused-beam technique has been described for determining the detailed characteristics of polydisperse systems of scatterers. In order to apply this technique it is necessary that the scattering properties of the individual particles be understood when the incident light is in the form of a focused Gaussian beam. Most previous treatments of scattering have regarded the light source as being a uniform plane wave, and we cannot a priori expect to obtain the same results with a focused beam. Recently the scattered field has been derived for a spherical dielectric particle at the axis of plane wave Gaussian beam for particles which are small compared to the beam diameter. ${ }^{7}$ A more general treatment has also been given which applies to on-axis particles of arbitrary size. ${ }^{8}$

As one would expect, the field distribution resulting with an incident Gaussian beam reduces to the familiar plane wave results in the limit that the beam diameter is much larger than the particle diameter. As an example, typical scattering patterns are reproduced in Fig. 2 for the case of a small conducting spherical particle. ${ }^{8}$ This figure shows the field distribution that results when a Gaussian beam of spot size $w_{o}=4.19 \lambda$ propagating in the $z$ direction and polarized in the $x$ direction is incident on a conducting sphere of radius $1.47 \lambda$. 


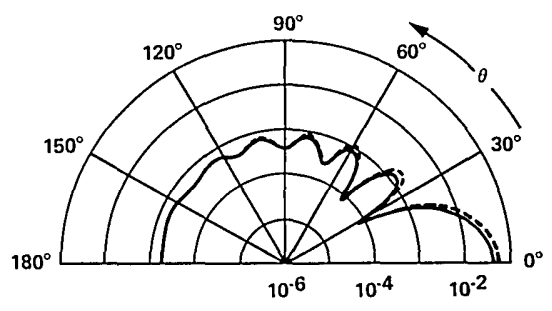

a.

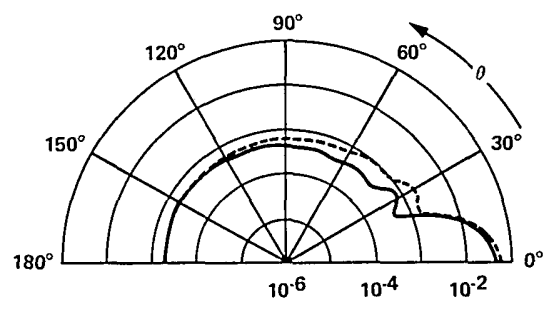

b.

Fig. 2. Scattered field distribution that results when a Gaussian beam of spot size $w_{1}=4.19 \lambda$ is incident on a conducting sphere of radius $a=1.47 \lambda$. Parts (a) and (b) show, respectively, the $\left.\left|E_{\ell}\right|^{2}\right|^{2}$ and $\left|E_{\phi}{ }^{r}\right|^{2}$ values of the scattered field with the exact results given by solid lines and the corresponding plane wave results given by dashed lines (after Ref. 8).

Parts (a) and (b) show, respectively, the $\left|E_{\theta}{ }^{r}\right|^{2}$ and $\left|E_{\phi}{ }^{r}\right|^{2}$ values of the scattered field with the exact results given by solid lines and the corresponding plane wave results given by dashed lines. $\left(E_{\theta}{ }^{r}\right.$ and $E_{\phi}{ }^{r}$ are, respectively, the $\theta$ component in the $x-z$ plane and the $\phi$ component in the $y-z$ plane.) These curves confirm the reasonable fact that for particles small compared to the beam diameter, the scattering pattern for a Gaussian beam is essentially identical to the more familiar plane wave scattering data.

For particles which are comparable in size to the beam diameter, the plane wave data cannot be employed. The previously obtained beam solutions ${ }^{7,8}$ are also not rigorously applicable in this case because of two complicating features of the problem. First, in our case the particles are not necessarily going to be at the axis of the light beam, and the scattering pattern will not be symmetric about the beam axis. Second, with a very low $f$-number focusing lens the phase fronts of the beam may be spherical rather than plane when they impinge upon the particle, and this fact will also affect the scattered fields. Because of the desirability of adopting the familiar plane wave scattering results, our initial experiments have employed focusing lenses with intermediate $f$-numbers and particles which are small compared to the beam diameter. More general theoretical models are in progress, so that in the future the above restrictions will not be necessary. Besides including off-axis spherical particles of arbitrary size and curved phase fronts of the incident beam, we are also adapting a previous analysis which accounts for scat- tering by particles of arbitrary shape. ${ }^{9}$ With these results it will be possible, for example, to distinguish a needle-shaped asbestos fiber in the atmosphere from other harmless particles of comparable volume.

\section{Experiment}

A general focused-beam technique has been described for determining the characteristics of an ensemble of scattering particles. We have confirmed the validity of this approach by means of experiments using particles of known properties, and the field distribution scattered from latex spheres is found to be in good agreement with the theoretical predictions. The experimental apparatus is shown schematically in Fig. 1. The linearly polarized $5145-\AA$ beam from an argon laser (Lexel model 75) is deflected downward into a cell containing the particles suspended in water. The cell has a spherical glass envelope $42 \mathrm{~mm}$ in diameter and is centered on the beam focus. This spherical shape is useful for eliminating refraction effects with liquidsuspended particles. Focusing has been accomplished by means of a microscope objective lens. The particles used in this study are latex spheres having a radius of $a=0.2405 \mu \mathrm{m}$ and a refractive index of $n=1.59$.

In an optimized configuration designed for investigating the properties of polydisperse systems of nonspherical particles, the detection scheme should consist of an array of detectors completely surrounding the scattering cell. Information from these detectors would be fed to an appropriate multichannel analysis system to determine the properties of the individual particles as they pass through the beam. Alternatively, the information could be carried from many points surrounding the cell to a remote detector array by means of optical fibers. However, our initial experiments have involved uniform dielectric spheres, and it has been sufficient to use a single detector which can be accurately scanned about the focus as shown in Fig. 1. Several events are recorded for each angular position, and the largest values correspond to particles near the focus. The weaker events result from particles crossing the beam away from the focus and are discarded. The off-focus events can also be largely eliminated if the detector is preceded by a spatial filter.

Typical experimental results obtained at intervals of $15^{\circ}$ are summarized in Fig. 3. The beam waist spot size is $w_{o}=0.54 \mu \mathrm{m}$, and each point represents approximately twenty measurements. The theoretical curve is a plot of $\left|E_{\theta}{ }^{r}\right|^{2}$ obtained from Lorenz-Mie scattering theory using the known refractive index of the particles together with the value $k a=3.6$. This latter number gives a slightly better fit than the theoretical value $k a$ $=2 \pi n a / \lambda=3.9$, and discrepancies of this order are not uncommon in detailed scattering studies. ${ }^{10}$ Measurements have also been made of the total power in the transmitted beam. When a particle passes through the focus, the transmitted power decreases, and the magnitude of this decrease can be used to determine the total scattering cross section. 


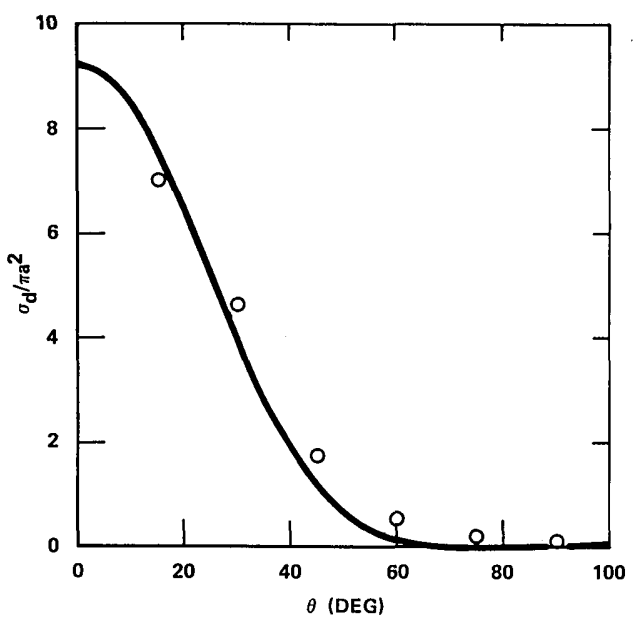

Fig. 3. Angular dependence of the differential scattering cross section $\sigma_{d}$ divided by the area $\pi a^{2}$. Solid line is exact result of Lorenz-

Mie theory, and points are experimental values.

\section{Conclusion}

A focused-beam scattering technique has been developed in which the effects of individual scatterers can be isolated without removing the scatterers from their natural environment. This technique is potentially advantageous for investigations of living organisms or particles which for any reason have characteristics that depend on the host medium. Experimental verification of the basic ideas has been obtained using monodisperse dielectric spheres. Extensions of these concepts will involve detailed scattering calculations and seeing to what extent a unique inversion is possible with more general systems of polydisperse and nonspherical particles.

This work was supported in part by the National Science Foundation.

\section{References}

1. M. Kerker, The Scattering of Light and Other Electromagnetic Radiation (Academic, New York, 1969).

2. C. Tanford, Physical Chemistry of Macromolecules (Wiley, New York, 1961).

3. Remote Measurement of Pollution (National Aeronautics and Space Administration, Washington, D.C., 1971).

4. D. T. Phillips, P. J. Wyatt, and R. M. Berkman, J. Colloid Interface Sci. 34, 159 (1970).

5. P. M. Morse, Thermal Physics (Benjamin, New York, 1965), Chap. 15.

6. Smithsonian Physical Tables, W. E. Forsythe, Ed. (Smithsonian, Washington, D.C., 1969), p. 319.

7. N. Morita, T. Tanaka, T. Yamasaki, and Y. Nakanishi, IEEE Trans. Antennas Propag. AP-16, 724 (1968).

8. W.-C. Tsai and R. J. Pogorzelski, J. Opt. Soc. Am. 65, 1457 (1975).

9. P. Barber and C. Yeh, Appl. Opt. 14, 2864 (1975).

10. R. G. Pinnick, D. E. Carroll, and D. J. Hofmann, Appl. Opt. 15, 384 (1976).

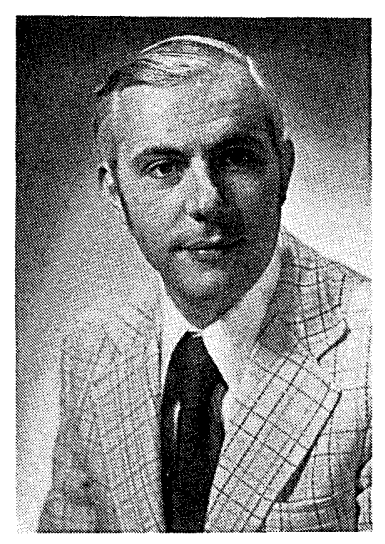

Simon L. Engel of GTE Sylvania's Industrial Lasers Applications Laboratories. 